\title{
Hydrological Analysis Method in Selecting Flood Discharge in Watershed of Kelara River
}

\author{
Muhammad Rifaldi Mustamin ${ }^{1, *}$, a, Farouk Maricar ${ }^{1}$, and Riswal Karamma ${ }^{1}$ \\ ${ }^{1}$ Civil Engineering Dept., Hasanuddin University, Jalan Perintis Kemerdekaan Km.10, Makassar, 90245, Indonesia \\ ${ }^{*}, \mathrm{a}$ aldirifal09@gmail.com (Corresponding Author)
}

\begin{abstract}
For Engineers involved in planning and construction of water resources building, hydrology becomes very important data. In terms of planning stage in water resources especially waterworks, it is known that design flood discharge closed to field realistic conditions is often needed in order that a planned construction is able to control flood discharge. Several previous researches in choosing flood discharge selection method have diverse depending on observed watershed. One method in determining selected flood discharge by verification using Creager diagram, by comparing discharge calculation results of several Synthetic Unit Hydrograph (SUH) with infrastructure flood discharge (AWLR result) in observation point. This research aims to obtain the most suitable synthetic unit hydrograph and close to analysis result of measured discharge frequency, and Creager diagram in Kelara watershed (DAS). Based on the calculation of design flood discharge according to rainfall data using synthetic unit hydrograph of Nakayasu, ITB I, ITB II, and SCS (HECHMS) as well as the calculation of design flood discharge according to collected data, it is concluded that the synthetic unit hydrograph method closest to design flood discharge with measured discharge rate and Q1000 rate of Creager diagram is SCS. Flood discharge rate obtained according to HSS SCS method using HEC-HMS 4.8 application in period of 2 years is 658,40 $\mathrm{m}^{3} / \mathrm{s}, 25$ years is $682,70 \mathrm{~m}^{3} / \mathrm{s}, 50$ years is $787,00 \mathrm{~m}^{3} / \mathrm{s}, 100$ years is $885,70 \mathrm{~m}^{3} / \mathrm{det}$, and 1000 years is $1202,60 \mathrm{~m}^{3} / \mathrm{s}$.
\end{abstract}

Keywords-Design Flood Discharge, Synthetic Unit Hydrograph, Measured Discharge Data, Kelara Watershed (DAS).

\section{Introduction}

In terms of hydrology, river functions to contain rainfall and flows it to the sea. An area where river first get the rainfall water is rain catchment area known as Watershed (DAS). It is so that Watershed can be seen as a unitary unit of rainfall area becoming river flow [1].

Critical data required in hydrological analysis are topographyy, rainfall rate, land use pattern, type of soil and the data collected from measured observations including water level and discharge rate of a watershed [2].

For engineers involved in design and construction stages of waterwork building, hydrological data is crucially needed. As an example, if a city is trying to improve or fix a water availability problem, then the first step to it for the engineer is to find out a watershed in highlands area and estimate its ability to provide water. On the other hand, engineers must also be able to predict potential flood in the watershed [3].

In water resource planning, especially in waterworks design, design flood discharge closed to the field realistic condition is often needed in order to design a building capable of controlling flood discharge. Design flood discharge per period can be calculated using actual flood discharge and rainfall data. If the actual discharge data is available then the design flood discharge can be directly calculated using probability analysis method. But, if rainfall data and the watershed characteristics data are the only data available, it is recommended to calculate flood discharge of daily maximum rainfall data using hydrograph [4].

In order to make hydrograph in a watershed in which the flood hydrograph observation data is either limited or unavailable, then it is needed to know the watershed characteristics before several approaches are carried out using methods developed by experts [5].

Several previous researches in selecting flood discharge method have different results depending on the reviewed watershed [6]-[10]. 
Although there have been many referable models, yet there are still doubts on how to apply the models in Indonesia with its tropical climate. This can be understood since the characteristics in tropical region varies between one area and another as well as the watershed response [11].

One of the ways in determining selected flood discharge can be done by verifying it using Creager diagram, by comparing discharge rate calculation of several Synthetic Unit Hydrograph methods with the infrastructure flood discharge of AWLR (Automatic Water Level Recorder on observed point [12].

In the Kelara watershed, there is Automatic Water Lever Recorder (AWLR) in the downstream area so that the research can be carried out to test discharge result of Synthetic Unit Hydrograph with AWLR frequency analysis of discharge rate.

\section{Research Methodology}

\section{A. Location}

The research is located in Kelara Watershed covering two regencies, Gowa Regency in upstream area dan Jeneponto Regency in downstream area. The Kelara Watershed belongs to the river basin of Jeneberang with researched area amounted to $288,62 \mathrm{~km}^{2}$.

\section{B. Data Collection Technique}

The data used in this research are:

1. Rainfall data of Rainfall station of Paitana and station of Tanrang. Rainfall data period of time used is ranged from 1999 - 2018 (20 years) obtained from Large River Basin Organization of Pompengan Jeneberang (BBWS Pompengan Jeneberang).

2. Measured discharge data from water-level measuring posts situated in research location, namely AWLR of Kelara in years of 1999 - 2018 obtained from River Basin Organization of Pompengan Jeneberang (BBWS Pompengan Jeneberang).

3. Soli classification map in 2018 obtained from River Basin Organization of Pompengan Jeneberang (BBWS Pompengan Jeneberang).
4. Land use pattern map in 2019 obtained from Geospatial Information Organization (BIG).

\section{Rainfall Data Analysis}

\section{Lost Data Entry}

In order to fill out the lost rainfall data caused by damaged devise/equipment or that the observer did not take notes in a station, it can be filled by estimating estimation value based on previous three stations surrounding. The filling of the lost rainfall data can use normal comparative method and reciprocal method [13]. In this research the method used in filling out the lost data is reciprocal method.

$$
P_{x}=\frac{\sum_{i=1}^{n} \frac{P i}{L i^{2}}}{\sum_{i=1}^{n} \frac{1}{L i^{2}}}
$$

Where Px is the lost rainfall data in station $\mathrm{x}(\mathrm{mm}), \mathrm{Pi}$ is rainfall data in surrounding stations in the same period of time $(\mathrm{mm})$ dan $\mathrm{Li}$ : Distance of station $\mathrm{x}$ and the surrounding stations $(\mathrm{km})$.

\section{Consistent Test}

Consistent test is carries out to view the provided data whether it is consistent or not so that it is feasible to use. Consisten test means to use the validity of field data not affected by the mistakes at the time of delivery or measurement. Data consistency test can be done by using Double Mass Curve method and Rescaled Adjusted Partial Sums (RAPS) [14].

In this research the used method in consistency test is Double Mass Curve method.

\section{Regional rainfall}

Regional rainfall (in units of $\mathrm{mm}$ ) is daily maximum rainfall in the whole region. Regional rainfall is needed in calculating design flood discharge [13]. The calculation of regional rainfall in this research used Thiessen Polygon method :

$$
\bar{p}=\frac{A_{1} p_{1}+A_{2} p_{2}+\ldots .+A_{n} p_{n}}{A_{1}+A_{2}+\ldots . .+A_{n}}
$$


Where $\mathrm{A}$ is the area representing each station.

\section{Frequency analysis}

In terms of rainfall frequency analysis or discharge data to obtain design rainfall value or design discharge, it is known that several continuous probability analysis distributions are often used, namely Gumbel, Normal, Log Normal, and Log Pearson Type III.

The determination of probability distribution suited to the data is carried out by matching the parameters like average value, deviation standard, drag coefficient (Cs), and kurtosis coefficient (Ck) [13].

5. Data distribution compatibility test

To find out whether the selected probability distribution equation is representable for analyzed data sample statistic distribution, probability distribution test is needed. The method used for the test are Chi-Square and Kolmogorov-Smirnov test [15].

\section{Rainfall Intensity}

Rainfall intensity is the ratio of the total amount of rain (rainfall depth) falling during a certain period to the duration of the period expressed in unit of $\mathrm{mm} / \mathrm{second}$. The using of the method for rainfall intensity calculation depending on the provided rainfall data. In this research as the provided rainfall data is in the daily period then Mononobe method is used [15].

\section{Net Rain Analysis}

Net rain in this research is calculated with SCS-CN method. The equation is as follows:

$$
\begin{gathered}
\mathrm{Pe}=(\mathrm{P}-\mathrm{Ia}) 2 /(\mathrm{P}-\mathrm{Ia}+\mathrm{S}) \\
\mathrm{S}=(25400 / \mathrm{CN})-254 \\
\mathrm{Ia}=0,2 \mathrm{~S}
\end{gathered}
$$

Where Pe is effective rain depth (mm), P is rain depth $(\mathrm{mm})$, Ia is initial abstraction $(\mathrm{mm}), \mathrm{S}$ is maximum potential water retention by soil mainly caused by infiltration ( $\mathrm{mm}$ ), and $\mathrm{CN}$ is constant value determined by type of soil [13].
Table 1. SCS Classification of Soil Hydrology Group According to Soil Texture.

\begin{tabular}{|c|c|c|c|}
\hline No. & Soil Texture & $\begin{array}{c}\text { Min. Infiltration } \\
\text { Rate(mm/jam) }\end{array}$ & $\begin{array}{c}\text { SCS Soil } \\
\text { Hydrology } \\
\text { Group }\end{array}$ \\
\hline 1 & Sand & 210 & A \\
\hline 2 & Loamy sand & 61 & A \\
\hline 3 & Sandy loam & 26 & B \\
\hline 4 & Loam & 13 & B \\
\hline 5 & Silty loam & 6,9 & C \\
\hline 6 & Sandy clay loam & 4,3 & C \\
\hline 7 & Silty clay loam & 2,3 & D \\
\hline 8 & Clay loam & 1,5 & D \\
\hline 9 & Sandy clay & 1,3 & D \\
\hline 10 & Silty clay & 1,0 & D \\
\hline 11 & Clay & 0,5 & D \\
\hline
\end{tabular}

Table 2. CN Value according to Land Use Pattern

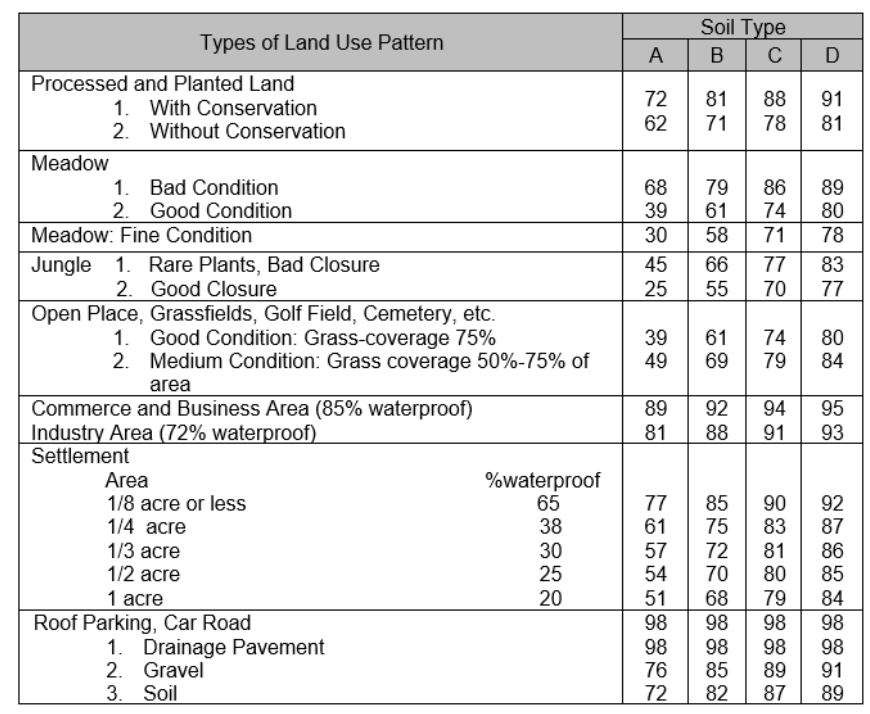

\section{Design Flood Discharge Analysis}

In this research design flood discharge analysis uses 4 method, Synthetic Unit Hydrograph of Nakayasu, ITB-I, ITB-II, and SCS (HEC-HMS). The following is the explanation of the four method :

\section{Nakayasu}

Nakayasu researched unit hydrograph in Japan and produced the equation as follows [13].

a. Lag time $\left(t_{g}\right)$

$$
\begin{gathered}
t_{g}=0,4+0,058 \times L(\text { for } \mathrm{L}>15 \mathrm{~km}) \\
t_{g}=0,21 \times L^{0,7}(\text { for } \mathrm{L}<15 \mathrm{~km})
\end{gathered}
$$


b. Peak time

$$
t_{p}=t_{g}+0,8 T r
$$

c. Discharge time is equal to 0,3 times peak discharge

$$
t_{0,3}=\alpha \times t_{g}
$$

d. Hydrograph peak discharge

$$
Q_{p}=\frac{1}{3,6} \times A \times R_{0} \times \frac{1}{\left(0,3 \times t_{p}+t_{0,3}\right)}
$$

Where $t_{\mathrm{g}}$ is the gap time (hour), $\mathrm{L}$ is river length $(\mathrm{km})$, $\mathrm{t}_{0,3}$ is discharge time equal to 0,3 kali peak (jam), $\mathrm{t}_{\mathrm{p}}$ is peak time (hours), $\alpha$ is coeffiecient with the value ranges from $1,5-2,0, Q_{p}$ is peak discharge $\left(\mathrm{m}^{3} / \mathrm{sec}\right), \mathrm{T}_{\mathrm{r}}$ is rainfall duration (hours), $R_{0}$ is rain depth unit ( $\mathrm{mm}$ ).

\section{ITB Synthetic Unit Hydrograph (HSS ITB)}

ITB Synthetic Unit Hydrograph, also called HSS ITBI was developed according to the experience of Dantje Kardana Natkusumah when doing evaluation toward design flood hydrograph in physical model test of two dam spillways in Citepus dan Sadawarna Dam [16].

The following is the equation used in ITB-I:

a. Lag time ( $\left.\mathrm{t}_{\mathrm{p}}\right)$

$$
t_{p}=C_{t} 0,81225 \cdot L^{0,6}
$$

b. Peak time

$$
T p=t_{p}+0,50 T r
$$

c. Unit hydrograph base time

$$
\mathrm{Tb}=(10 \mathrm{~s} / \mathrm{d} 20) \mathrm{Tp}
$$

d. Hydrograph peak discharge

$$
Q p=\frac{R}{3,6 T p} \frac{A_{D A S}}{A_{H S S}}
$$

The following is the equation used in ITB-II :

a. Lag time $\left(t_{p}\right)$

$$
\begin{aligned}
t_{p} & =C_{t} \times(0,527+0,058 \cdot L)(\text { untuk L }>15 \mathrm{~km}) \\
t_{p} & =C_{t} \times\left(0,21 \cdot L^{0,7}\right)(\text { untuk } \mathrm{L}<15 \mathrm{~km})
\end{aligned}
$$

b. Peak time

$$
T p=t_{p}+0,60 t_{p}
$$

The equation of unit hydrograph base time and peak discharge is the same as ITB I.

Where tp is Lag time (hours), $\mathrm{L}$ is river length $(\mathrm{km})$, $\mathrm{C}_{\mathrm{t}}$ Calibration coefficient $=1, \mathrm{~T}_{\mathrm{r}}$ is rain duration (hours), $\mathrm{Tp}$ is peak time (hours), Tb unit hydrograph base time, $\mathrm{Q}_{\mathrm{p}}$ is peak discharge rate $\left(\mathrm{m}^{3} / \mathrm{det}\right), \mathrm{R}$ is unit rainfall rate $(\mathrm{mm}), \mathrm{A}_{\mathrm{DAS}}$ is watershed area value $\left(\mathrm{km}^{2}\right)$, and $A_{H S S}$ is dimensionless unit hydrograph curve area.

\section{SCS Synthetic Unit Hydrograph (HEC-HMS)}

SCS Synthetic Unit Hydrograph is dimensionless unit hydrograph, where this method calculates the influence of soil type and land use pattern and can be calculated using HEC-HMS software. The following is the equation used in SCS method [17] :

a. Hydrograph peak discharge and peak time

$$
\begin{gathered}
U_{p}=C \frac{A}{T_{p}} \\
T_{p}=\frac{\Delta t}{2}+t_{\text {lag }}
\end{gathered}
$$

b. Lag time $\left(t_{p}\right)$

$$
t_{p}=0,6 \cdot t_{c}
$$

c. Kirpich time of concentration

$$
t_{c}=\left(\frac{0,87 \times L^{2}}{1000 \times S}\right)^{0,385}
$$

Where Up is peak discharge, A is watershed area, Tp is peak time, $\mathrm{C}$ is constant value $(2,08), \Delta \mathrm{t}$ is period of excess rain, tp is Lag time (hours), tc is time of concentration, $\mathrm{L}$ is river length $(\mathrm{km})$, dan $\mathrm{S}$ is river slope $(\mathrm{m} / \mathrm{m})$.

\section{E. Research flowchart}

Generally, research flowchart can be seen in Fig. 1. 


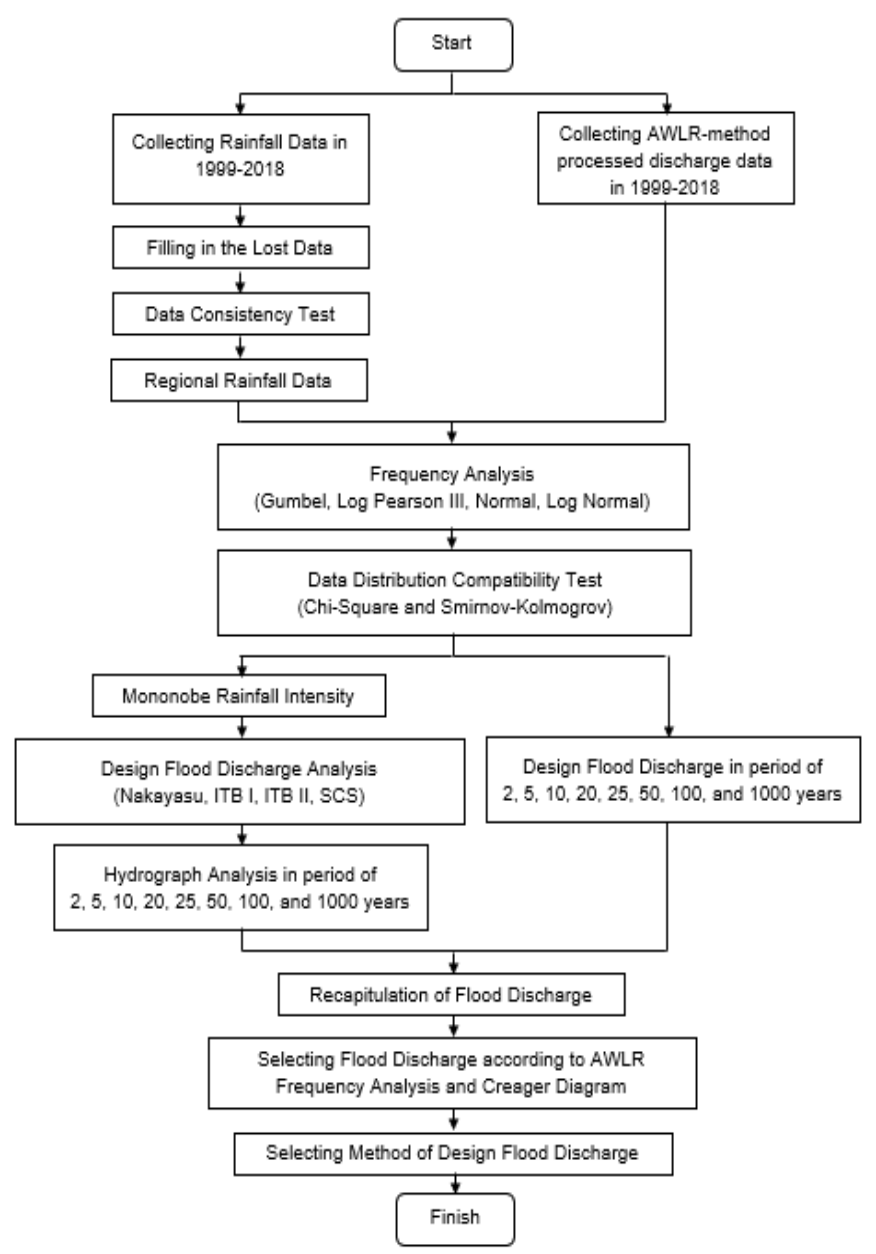

Figure 1. Research Stages

\section{Results and Discussion}

\section{A. Characteristic Analysis of Kelara sub-watershed}

The map of Kelara Sub-Watershed is needed in order to obtain the characteristics of the watershed like area, main river length, slope incline, and Sub-Watershed boundaries needed in analysis process. The following is the map of the watershed, soil type, and land use pattern of research location.

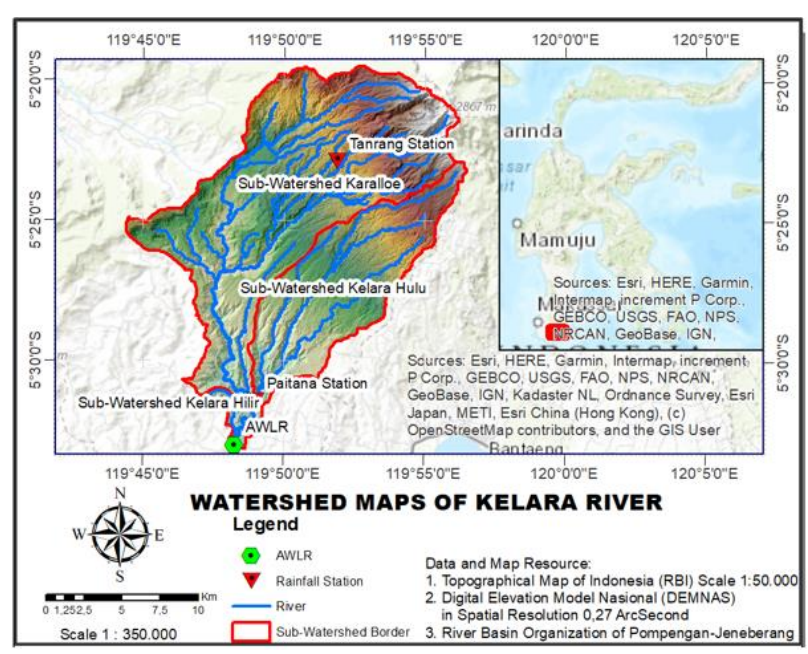

Figure 2. Map of Kelara Watershed

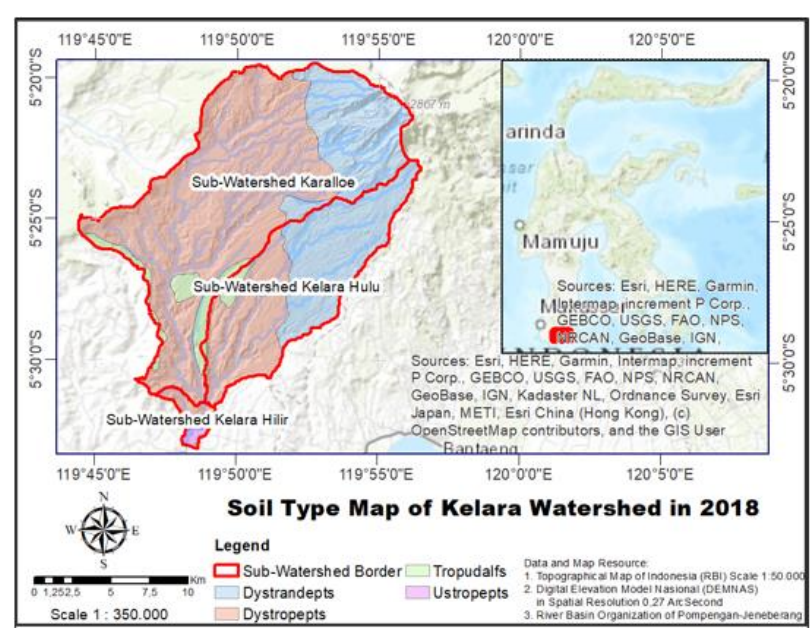

Figure 3. Soil Type Map of Kelara Watershed

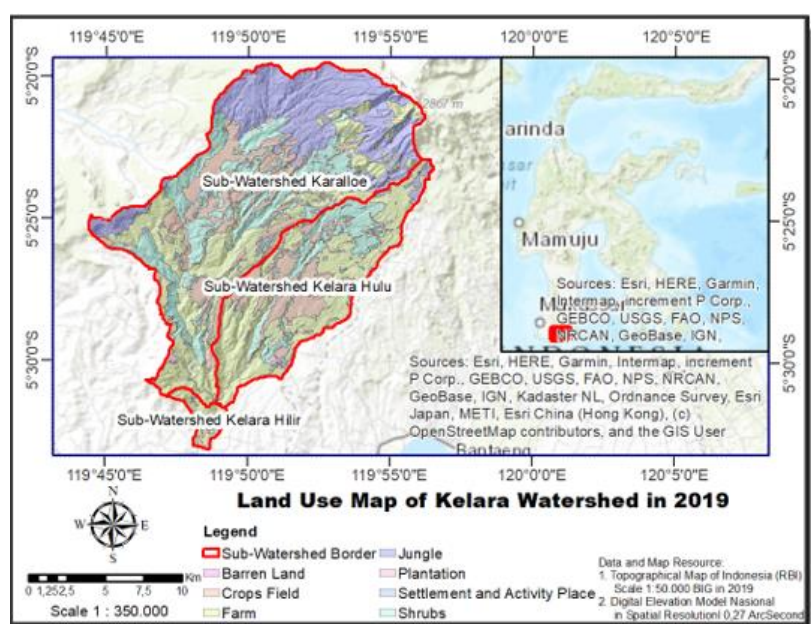

Figure 4. Land Use Pattern Map of Kelara Watershed 
Table 3. Characteristics Recapitulation of Kelara Sub-Watershed

\begin{tabular}{|c|c|c|c|c|}
\hline \multirow[b]{2}{*}{ No. } & \multirow{2}{*}{$\begin{array}{l}\text { Physical } \\
\text { Parameter }\end{array}$} & \multicolumn{3}{|c|}{ Sub-Watershed } \\
\hline & & Karalloe & $\begin{array}{c}\text { Kelara } \\
\text { Hulu }\end{array}$ & $\begin{array}{c}\text { Kelara } \\
\text { Hilir }\end{array}$ \\
\hline 1 & Area $\left(\mathrm{km}^{2}\right)$ & 195,23 & 86,89 & 6,52 \\
\hline 2 & $\begin{array}{l}\text { River Length } \\
(\mathrm{km})\end{array}$ & 27,27 & 12,62 & 5,38 \\
\hline 3 & $\begin{array}{c}\text { Upstream } \\
\text { Elevation }(\mathrm{msl})\end{array}$ & 848,00 & 753,42 & 140,13 \\
\hline 4 & $\begin{array}{c}\text { Downstream } \\
\text { Elevation }(\mathrm{msl})\end{array}$ & 140,13 & 140,13 & 99,87 \\
\hline 5 & River Slope & 0,026 & 0,049 & 0,007 \\
\hline 6 & $\begin{array}{l}\text { Max. Potential } \\
\text { Retention (mm) }\end{array}$ & 117,01 & 81,67 & 111,19 \\
\hline 7 & $\begin{array}{c}\text { Initial } \\
\text { Abstraction } \\
(\mathrm{mm}) \\
\end{array}$ & 23,40 & 16,33 & 22,24 \\
\hline 8 & Imoervious (\%) & 0,58 & 0,93 & 0,59 \\
\hline 9 & $\begin{array}{l}\text { Curve Number } \\
\text { (CN) }\end{array}$ & 68 & 76 & 70 \\
\hline 10 & Lag Time (min) & 124,17 & 53,887 & 57,41 \\
\hline
\end{tabular}

\section{B. Yearly Maximum Rainfall Analysis}

Design rain is needed in calculation of design flood discharge in which the value is obtained from yearly maximum daily rainfall analysis. The data range provided in rainfall station of Kelara Watershed is in period of 20 years starting from 1999 until 2018. The lost rainfall data or the uncompleted one is complemented by using reciprocal method. The uncompleted one is in Paitana station in years of 2004-2007. Yearly Maximum Daily Rainfall Data which is influential is tabled in Table 4.

Table 4. Kelara Watershed Max. Daily Rainfall

\begin{tabular}{|c|c|c|c|}
\hline \multirow{2}{*}{ No. } & \multirow{2}{*}{ Year } & \multicolumn{2}{|c|}{ Max. Daily Rainfall (mm) } \\
\cline { 3 - 4 } & & Paitana Sta. & Tanrang Sta. \\
\hline 1 & 1999 & 90 & 90 \\
\hline 2 & 2000 & 90 & 95 \\
\hline 3 & 2001 & 75 & 80 \\
\hline 4 & 2002 & 100 & 80 \\
\hline 5 & 2003 & 75 & 100 \\
\hline 6 & 2004 & 135 & 45 \\
\hline 7 & 2005 & 39 & 68 \\
\hline 8 & 2006 & 131 & 125 \\
\hline 9 & 2007 & 68 & 90 \\
\hline 10 & 2008 & 73 & 136 \\
\hline 11 & 2009 & 90 & 92 \\
\hline 12 & 2010 & 95 & 91 \\
\hline 13 & 2011 & 77 & 51 \\
\hline 14 & 2012 & 65 & 94 \\
\hline 15 & 2013 & 85 & 125 \\
\hline 16 & 2014 & 80 & 125 \\
\hline 17 & 2015 & 80 & 85 \\
\hline 18 & 2016 & 95 & 79 \\
\hline
\end{tabular}

\begin{tabular}{|c|c|c|c|}
\hline \multirow{2}{*}{ No. } & \multirow{2}{*}{ Year } & \multicolumn{2}{|c|}{ Max. Daily Rainfall (mm) } \\
\cline { 3 - 4 } & & Paitana Sta. & Tanrang Sta. \\
\hline 19 & 2017 & 75 & 75 \\
\hline 20 & 2018 & 75 & 105 \\
\hline
\end{tabular}

\section{Data Consistency Test}

It is known that to evaluate the consistency in data series, consistency test is used. The method used in this test is Double Mass Curve Method.

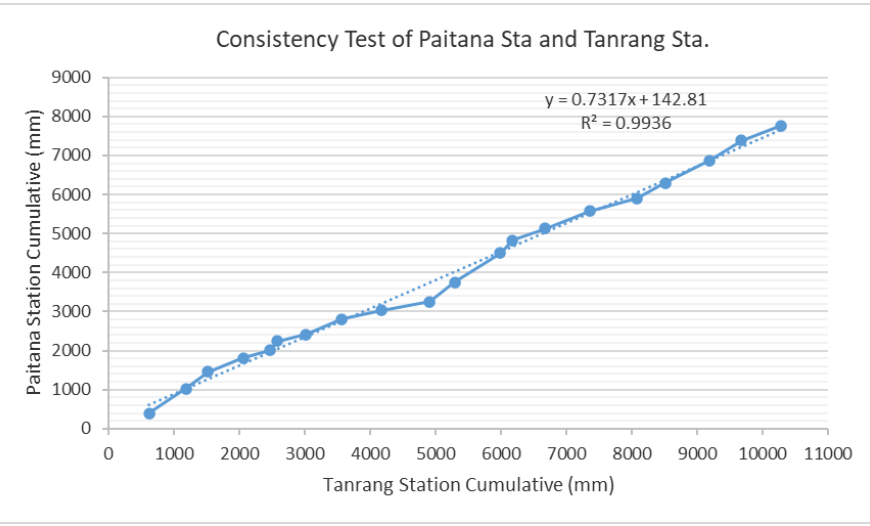

Figure 5. Consistency Test Result of Paitana Sta. and Tanrang Sta.

From the consistency test of yearly rainfall data, each station shows that the data is consistent enough with correlation value amounted to $99,36 \%$.

\section{Regional Rainfall}

This analysis is needed to know the influence of rainfall station toward a watershed. This analysis will result in obtaining Thiessen Coefficient which will be multiplied by each station. Thiessen coeff, value on Kelara Watershed can be seen in Table 5.

Table 5. Thiessen Coeff. of Kelara Watershed

\begin{tabular}{|c|c|c|c|}
\hline \multirow{2}{*}{ No. } & \multirow{2}{*}{ Sub-Watershed } & \multicolumn{2}{|c|}{ Thiessen Coeff. (mm) } \\
\cline { 3 - 4 } & & Paitana Sta. & Tanrang Sta. \\
\hline 1 & Karalloe & 0,21 & 0,79 \\
\hline 2 & Kelara Hulu & 0,45 & 0,55 \\
\hline 3 & Kelara Hilir & 1 & - \\
\hline
\end{tabular}

\section{E. Frequency Analysis}

There are 4 methods used in frequency analysis i.e. Normal Distribution, Log Normal, Gumbel, dan Log Pearson III.

Table 6. Frequency Analysis Sub-Watershed Karalloe 


\begin{tabular}{|c|c|c|c|c|c|}
\hline \multirow{2}{*}{ No. } & Period & $\begin{array}{c}\text { Log } \\
\text { Pearson } \\
\text { III }\end{array}$ & $\begin{array}{c}\text { Gumbel } \\
\text { Tipe I }\end{array}$ & Normal & $\begin{array}{c}\text { Normal } \\
\text { Log }\end{array}$ \\
\cline { 2 - 6 } & (yrs) & $(\mathrm{mm})$ & $(\mathrm{mm})$ & $(\mathrm{mm})$ & $(\mathrm{mm})$ \\
\hline 1 & 2 & 89.012 & 87.349 & 90.172 & 88.237 \\
\hline 2 & 5 & 106.010 & 107.722 & 106.220 & 105.751 \\
\hline 3 & 10 & 115.578 & 121.212 & 114.626 & 116.273 \\
\hline 4 & 20 & 124.441 & 134.151 & 121.503 & 125.655 \\
\hline 5 & 25 & 126.293 & 138.255 & 122.809 & 127.519 \\
\hline 6 & 50 & 133.486 & 150.899 & 129.336 & 137.266 \\
\hline 7 & 100 & 140.106 & 163.450 & 134.685 & 145.806 \\
\hline 8 & 1000 & 159.616 & 204.921 & 149.204 & 171.760 \\
\hline
\end{tabular}

Table 7. Frequency Analysis of Sub Watershed Kelara Hulu

\begin{tabular}{|c|c|c|c|c|c|}
\hline \multirow{2}{*}{ No. } & Period & $\begin{array}{c}\text { Log } \\
\text { Pearson } \\
\text { III }\end{array}$ & $\begin{array}{c}\text { Gumbel } \\
\text { Tipe I }\end{array}$ & Normal & $\begin{array}{c}\text { Normal } \\
\text { Log }\end{array}$ \\
\cline { 2 - 6 } & $(\mathrm{yrs})$ & $(\mathrm{mm})$ & $(\mathrm{mm})$ & $(\mathrm{mm})$ & $(\mathrm{mm})$ \\
\hline 1 & 2 & 88.183 & 86.261 & 88.584 & 87.251 \\
\hline 2 & 5 & 101.774 & 103.026 & 101.790 & 101.515 \\
\hline 3 & 10 & 109.050 & 114.126 & 108.707 & 109.895 \\
\hline 4 & 20 & 115.545 & 124.774 & 114.366 & 117.263 \\
\hline 5 & 25 & 116.890 & 128.151 & 115.440 & 118.716 \\
\hline 6 & 50 & 121.979 & 138.556 & 120.812 & 126.258 \\
\hline 7 & 100 & 126.534 & 148.884 & 125.213 & 132.794 \\
\hline 8 & 1000 & 139.406 & 183.010 & 137.161 & 152.292 \\
\hline
\end{tabular}

Table 8. Analisis Frekuensi Sub DAS Kelara Hilir

\begin{tabular}{|c|c|c|c|c|c|}
\hline \multirow{2}{*}{ No. } & Period & $\begin{array}{c}\text { Log } \\
\text { Pearson } \\
\text { III }\end{array}$ & $\begin{array}{c}\text { Gumbel } \\
\text { Tipe I }\end{array}$ & Normal & $\begin{array}{c}\text { Normal } \\
\text { Log }\end{array}$ \\
\cline { 2 - 6 } & $(\mathrm{yrs})$ & $(\mathrm{mm})$ & $(\mathrm{mm})$ & $(\mathrm{mm})$ & $(\mathrm{mm})$ \\
\hline 1 & 2 & 83.203 & 81.878 & 84.938 & 82.653 \\
\hline 2 & 5 & 101.273 & 103.955 & 102.327 & 101.144 \\
\hline 3 & 10 & 111.943 & 118.571 & 111.435 & 112.427 \\
\hline 4 & 20 & 122.014 & 132.591 & 118.887 & 122.589 \\
\hline 5 & 25 & 124.135 & 137.039 & 120.302 & 124.619 \\
\hline 6 & 50 & 132.503 & 150.740 & 127.374 & 135.285 \\
\hline 7 & 100 & 140.351 & 164.339 & 133.171 & 144.703 \\
\hline 8 & 1000 & 164.251 & 209.275 & 148.903 & 173.704 \\
\hline
\end{tabular}

\section{F. Data Distribution Compatibility Test}

This test is meant to know hypothesis truth of frequency distribution. There are 2 ways to carry out the test i.e. Chi-Square and Smirnov-Kolmogorov Test. The following is the result test on Kelara Watershed:
Table 9. Data Distribution Compatibility Test Sub-Watershed Karalloe

\begin{tabular}{|c|c|c|c|c|c|}
\hline \multirow{3}{*}{ No. } & \multirow{3}{*}{ Method } & \multicolumn{4}{|c|}{ Compatibility Test } \\
\hline & & \multicolumn{2}{|c|}{ Chi-Square } & \multicolumn{2}{|c|}{$\begin{array}{c}\text { Smirnov - } \\
\text { Kolmogorov }\end{array}$} \\
\hline & & $\chi^{2}$ hit & $\chi^{2} \mathrm{kr}$ & $\Delta_{\text {hit. }}$ & $\Delta_{\mathrm{kr}}$ \\
\hline \multirow{2}{*}{1} & \multirow{2}{*}{ Gumbel } & 4,6000 & 7,8150 & 0,1143 & 0,2940 \\
\hline & & \multicolumn{2}{|c|}{ Qualified } & \multicolumn{2}{|c|}{ Qualified } \\
\hline \multirow{2}{*}{2} & \multirow{2}{*}{ Log Pearson III } & 4,000 & 7,8150 & 0,1063 & 0,2940 \\
\hline & & \multicolumn{2}{|c|}{ Qualified } & \multicolumn{2}{|c|}{ Qualified } \\
\hline \multirow{2}{*}{3} & \multirow{2}{*}{ Normal } & 4,600 & 7,8150 & 0,1195 & 0,2940 \\
\hline & & \multicolumn{2}{|c|}{ Qualified } & \multicolumn{2}{|c|}{ Qualified } \\
\hline \multirow{2}{*}{4} & \multirow{2}{*}{ Log Normal } & 4,000 & 7,8150 & 0,0888 & 0,2940 \\
\hline & & \multicolumn{2}{|c|}{ Qualified } & \multicolumn{2}{|c|}{ Qualified } \\
\hline
\end{tabular}

With the same way, it is done to Sub-Watershed Kelara Hulu and Kelara Hilir. Chi - Square and SmirnovKolmogorov test shows that design daily rainfall distribution of Sub-Watershed Karalloe and Kelara Hilir follows Log Normal frequency distribution, and Sub Watershed Kelara Hulu follows Gumbel distribution.

\section{G. Rainfall Intensity and Net Rainfall}

Observation in Indonesia shows that centered rain do not last more than 7 hours, then in this calculation it is assumed that maximum centered rain period is 6 hours a day. Hourly rainfall distribution is calculated using Mononobe equation and Net rainfall analysis using SCS$\mathrm{CN}$ method which can be seen in following Table.

Table 10. Net Rainfall Calculation Sub-Watershed Karalloe

\begin{tabular}{|c|c|c|c|c|c|}
\hline Period & $\begin{array}{c}\text { Design } \\
\text { Rainfall }\end{array}$ & $\begin{array}{c}\text { Curve } \\
\text { Number }\end{array}$ & $\begin{array}{c}\text { Potential } \\
\text { Retention }\end{array}$ & $\begin{array}{c}\text { Initial } \\
\text { Abstraction }\end{array}$ & $\begin{array}{c}\text { Net } \\
\text { Rain }\end{array}$ \\
\hline$(\mathrm{yrs})$ & $(\mathrm{mm})$ & & $(\mathrm{mm})$ & $(\mathrm{mm})$ & $(\mathrm{mm})$ \\
\hline 2 & 88.24 & 68.46 & 117.01 & 23.40 & 23.12 \\
\hline 5 & 105.75 & 68.46 & 117.01 & 23.40 & 34.02 \\
\hline 10 & 116.27 & 68.46 & 117.01 & 23.40 & 41.10 \\
\hline 20 & 125.65 & 68.46 & 117.01 & 23.40 & 47.69 \\
\hline 25 & 127.52 & 68.46 & 117.01 & 23.40 & 49.02 \\
\hline 50 & 137.27 & 68.46 & 117.01 & 23.40 & 56.16 \\
\hline 100 & 145.81 & 68.46 & 117.01 & 23.40 & 62.58 \\
\hline 1000 & 171.76 & 68.46 & 117.01 & 23.40 & 82.94 \\
\hline
\end{tabular}


Table 11. Calculation of Effective Rainfall Sub-Watershed Karalloe

\begin{tabular}{|c|c|c|c|c|c|c|c|c|c|}
\hline \multirow{3}{*}{$\mathrm{t}$} & \multirow[b]{2}{*}{ Rt } & \multicolumn{8}{|c|}{ Net Raifall $(\mathrm{Rn}, \mathrm{mm})$ in Certain Period (years) } \\
\hline & & 2 & 5 & 10 & 20 & 25 & 50 & 100 & 1000 \\
\hline & & 23.12 & 34.02 & 41.10 & 47.69 & 49.02 & 56.16 & 62.58 & 82.94 \\
\hline (hour) & $(\%)$ & \multicolumn{8}{|c|}{ Hourly Net Rainfall = Rn x Rt } \\
\hline 1 & $55.03 \%$ & 12.72 & 18.72 & 22.62 & 26.24 & 26.98 & 30.90 & 34.44 & 45.65 \\
\hline 2 & $14.30 \%$ & 3.31 & 4.87 & 5.88 & 6.82 & 7.01 & 8.03 & 8.95 & 11.86 \\
\hline 3 & $10.03 \%$ & 2.32 & 3.41 & 4.12 & 4.78 & 4.92 & 5.63 & 6.28 & 8.32 \\
\hline 4 & $7.99 \%$ & 1.85 & 2.72 & 3.28 & 3.81 & 3.92 & 4.49 & 5.00 & 6.63 \\
\hline 5 & $6.75 \%$ & 1.56 & 2.29 & 2.77 & 3.22 & 3.31 & 3.79 & 4.22 & 5.59 \\
\hline 6 & $5.90 \%$ & 1.36 & 2.01 & 2.42 & 2.81 & 2.89 & 3.31 & 3.69 & 4.89 \\
\hline
\end{tabular}

Table 12. Calculation of Net Rainfall Sub-Watershed Kelara Hulu

\begin{tabular}{|c|c|c|c|c|c|}
\hline Period & $\begin{array}{c}\text { Design } \\
\text { Rainfall }\end{array}$ & $\begin{array}{c}\text { Curve } \\
\text { Number }\end{array}$ & $\begin{array}{c}\text { Potential } \\
\text { Retention }\end{array}$ & $\begin{array}{c}\text { Initial } \\
\text { Abstraction }\end{array}$ & $\begin{array}{c}\text { Net } \\
\text { Rain }\end{array}$ \\
\hline$(\mathrm{yrs})$ & $(\mathrm{mm})$ & & $(\mathrm{mm})$ & $(\mathrm{mm})$ & $(\mathrm{mm})$ \\
\hline 2 & 86.26 & 75.67 & 81.67 & 16.33 & 32.26 \\
\hline 5 & 103.03 & 75.67 & 81.67 & 16.33 & 44.64 \\
\hline 10 & 114.13 & 75.67 & 81.67 & 16.33 & 53.29 \\
\hline 20 & 124.77 & 75.67 & 81.67 & 16.33 & 61.86 \\
\hline 25 & 128.15 & 75.67 & 81.67 & 16.33 & 64.62 \\
\hline 50 & 138.56 & 75.67 & 81.67 & 16.33 & 73.27 \\
\hline 100 & 148.88 & 75.67 & 81.67 & 16.33 & 82.02 \\
\hline 1000 & 183.01 & 75.67 & 81.67 & 16.33 & 111.8 \\
\hline
\end{tabular}

Table 13. Calculation of Effective Rainfall Sub-Watershed Kelara Hulu

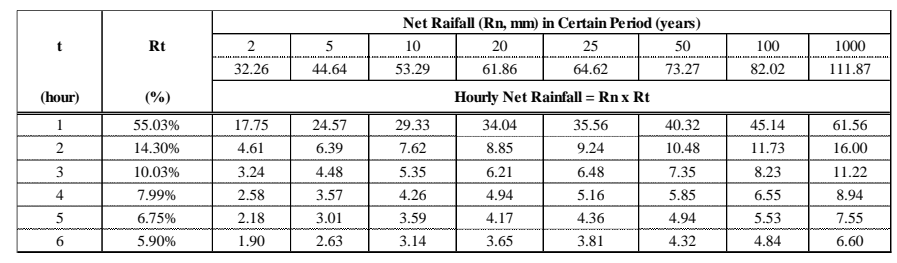

Table 14. Calculation of Net Rainfall Sub-Watershed Kelara Hilir

\begin{tabular}{|c|c|c|c|c|c|}
\hline Period & $\begin{array}{c}\text { Design } \\
\text { Rainfall }\end{array}$ & $\begin{array}{c}\text { Curve } \\
\text { Number }\end{array}$ & $\begin{array}{c}\text { Potential } \\
\text { Retention }\end{array}$ & $\begin{array}{c}\text { Initial } \\
\text { Abstraction }\end{array}$ & $\begin{array}{c}\text { Net } \\
\text { Rain }\end{array}$ \\
\hline$(\mathrm{yrs})$ & $(\mathrm{mm})$ & & $(\mathrm{mm})$ & $(\mathrm{mm})$ & $(\mathrm{mm})$ \\
\hline 2 & 82.65 & 69.55 & 111.19 & 22.24 & 21.27 \\
\hline 5 & 101.14 & 69.55 & 111.19 & 22.24 & 32.75 \\
\hline 10 & 112.43 & 69.55 & 111.19 & 22.24 & 40.39 \\
\hline 20 & 122.59 & 69.55 & 111.19 & 22.24 & 47.60 \\
\hline 25 & 124.62 & 69.55 & 111.19 & 22.24 & 49.08 \\
\hline 50 & 135.28 & 69.55 & 111.19 & 22.24 & 56.99 \\
\hline 100 & 144.70 & 69.55 & 111.19 & 22.24 & 64.19 \\
\hline 1000 & 173.70 & 69.55 & 111.19 & 22.24 & 87.34 \\
\hline
\end{tabular}

Table 15. Calculation of Effective Rainfall Sub-Watershed Kelara Hilir

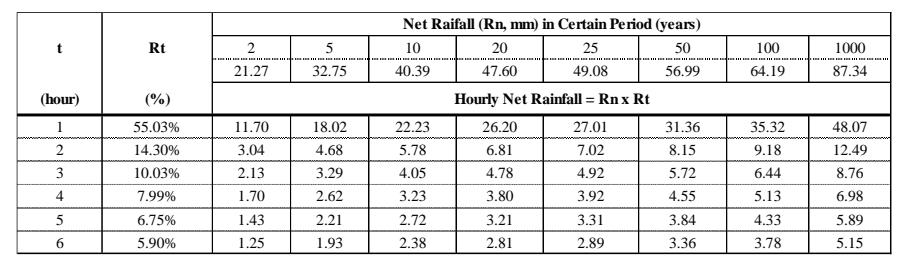

\section{H. Design Flood Discharge Analysis}

Design flood discharge is carried out in 4 method i.e. Synthetic Unit Hydrograph of Nakayasu, ITB I, ITB II and SCS (HEC-HMS). The result in this research is the result of superposition of the three Sub-Watershed to obtain total design flood discharge of Kelara Watershed located in outlet location of AWLR Kelara. The following is the superposition result of three Sub-Watershed in the four methods:

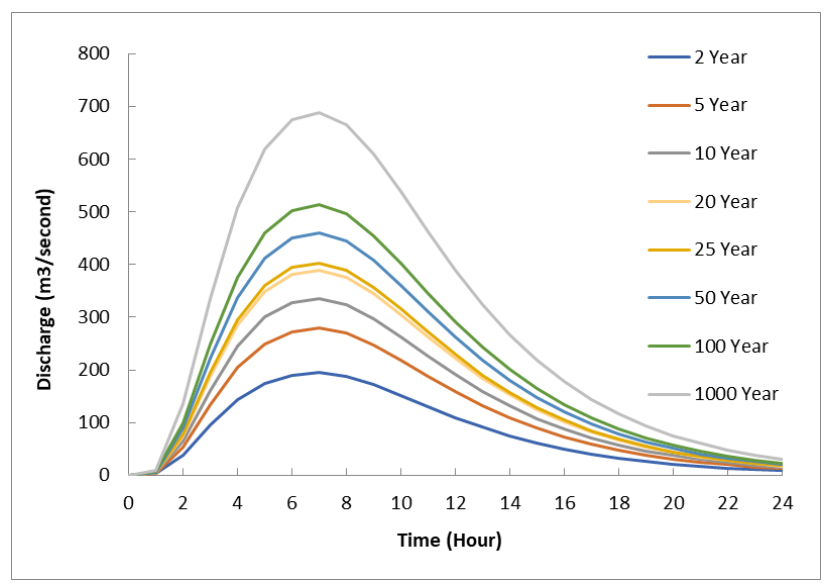

Figure 6. ITB I, Kelara Watershed

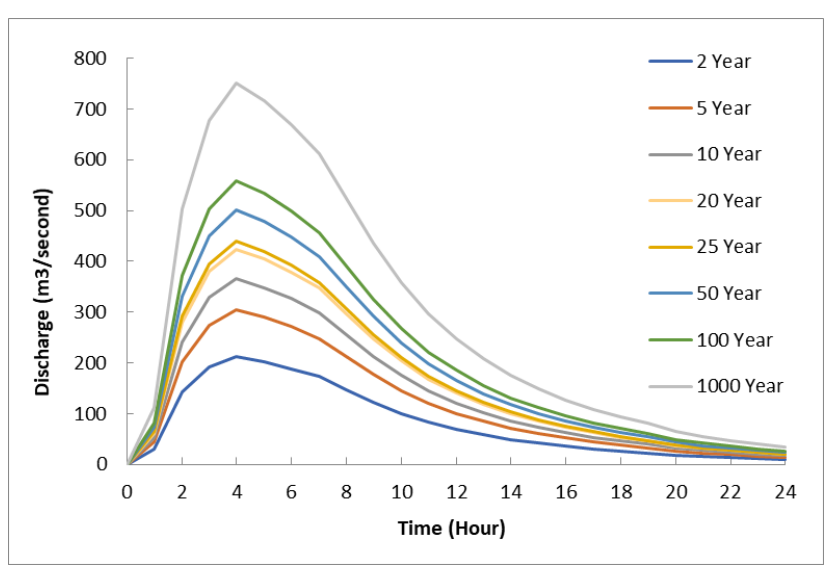

Figure 7. ITB II, Kelara Watershed

\section{Discharge Data Frequency Analysis}

Maximum river discharge data used in this analysis is in period of 1999 - 2018 from AWLR processed by River Basin Organization of Pompengan Jeneberang (BBWS Pompengan Jeneberang) as seen in Table 16. 


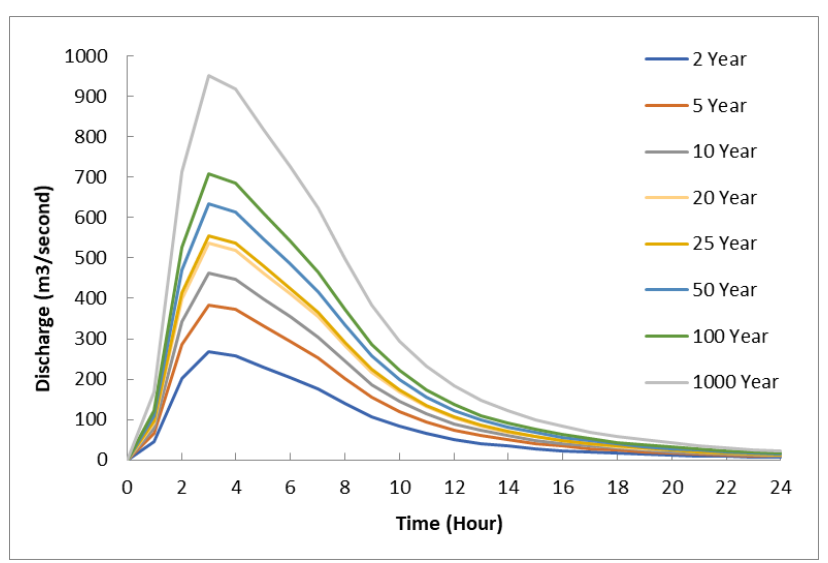

Figure 8. Nakayasu, Kelara Watershed

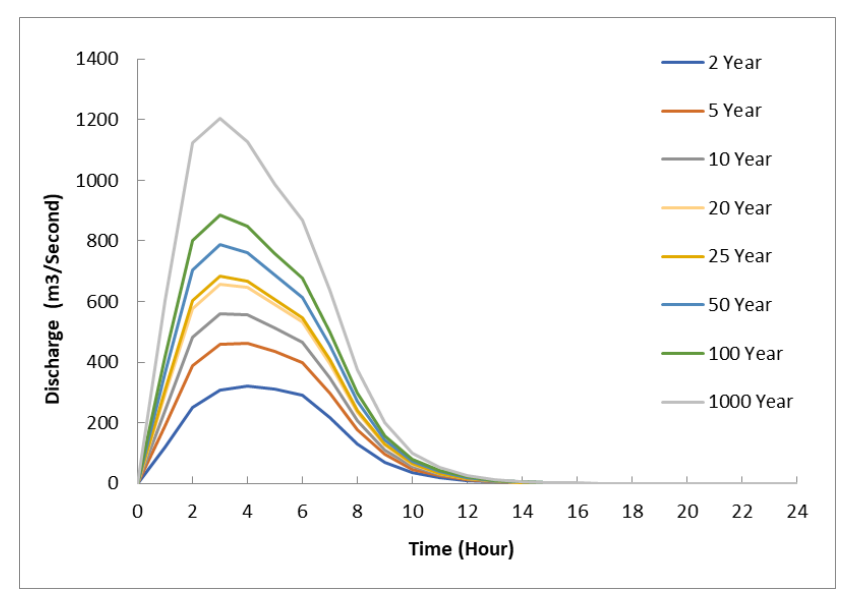

Figure 9. SCS (HEC-HMS), Kelara Watershed

Table 16. Maximum Discharge Rate of Kelara Watershed

\begin{tabular}{|c|c|c|}
\hline No. & Year & $\begin{array}{c}\text { Max. Discharge Rate of Kelara River } \\
\left(\mathrm{m}^{3} / \mathrm{sec}\right)\end{array}$ \\
\hline 1 & 1999 & 111.16 \\
\hline 2 & 2000 & 121.30 \\
\hline 3 & 2001 & 76.14 \\
\hline 4 & 2002 & 156.35 \\
\hline 5 & 2003 & 142.36 \\
\hline 6 & 2004 & 158.09 \\
\hline 7 & 2005 & 87.53 \\
\hline 8 & 2006 & 51.14 \\
\hline 9 & 2007 & 21.48 \\
\hline 10 & 2008 & 7.86 \\
\hline 11 & 2009 & 8.68 \\
\hline 12 & 2010 & 25.34 \\
\hline 13 & 2011 & 4.89 \\
\hline 14 & 2014 & 20.70 \\
\hline 15 & 2016 & 39.73 \\
\hline 16 & 2017 & 67.89 \\
\hline 17 & 2018 & 45.29 \\
\hline
\end{tabular}

From measured discharge data frequency analysis uses normal distribution, log normal distribution, gumbel distribution and log pearson type III distribution and distribution compatibility test using Chi-Square Test and Smirnov-Kolmogorov Test it is concluded that the design flood discharge as follows in Table 17.

Table 17. Design Flood Discharge according to Measured

\begin{tabular}{|c|c|c|}
\hline \multirow{2}{*}{ No. } & Period & Flood Discharge \\
\cline { 2 - 3 } & $(\mathrm{yrs})$ & $\left(\mathrm{m}^{3} / \mathrm{sec}\right)$ \\
\hline 1 & 2 & 43.597 \\
\hline 2 & 5 & 109.728 \\
\hline 3 & 10 & 177.948 \\
\hline 4 & 20 & 264.297 \\
\hline 5 & 25 & 284.906 \\
\hline 6 & 50 & 414.717 \\
\hline 7 & 100 & 564.122 \\
\hline 8 & 1000 & 1300.345 \\
\hline
\end{tabular}

J. Selection of Design Flood Discharge according to Measured Discharge and Creager Diagram

It is given that to determine method of selected design flood discharge can be controlled using Measured Discharge and Creager Diagram.

Creager diagram is used to compare design flood discharge Q in 1000-years period, in which the closest one to it according to Creager diagram is selected.

Value of $\mathrm{C}=100$ is used to calculate peak discharge of the biggest potential flood only happens once for the relevant river lifespan and various experiences show that the numbers are realistic [18].

According to above idea, it can be conluded that Creager coefficient used is $\mathrm{C}=100$. Creager diagram can be seen in Figure 10. According to Figure 10 shows that 1000 -years period viewed by all four methods the closest one to $\mathrm{C}=100$ of Creager diagram and measured discharge is SCS (HEC - HMS) and to optimize the river waterworks building design performance considering the discharge produced of SCS method (HEC - HMS) is greater than compared to another method.

Table 18. Design Flood Discharge Recapitulation

\begin{tabular}{|c|c|c|c|c|c|}
\hline \multirow{2}{*}{$\begin{array}{c}\text { Period } \\
(\text { Tr })\end{array}$} & $\begin{array}{c}\text { AWLR } \\
\text { Frequency } \\
\text { Analysis }\end{array}$ & SCS & Nakayasu & ITB I & ITB II \\
\hline 2 & 43.60 & 322.70 & 268.52 & 194.35 & 211.76 \\
\hline 5 & 109.73 & 464.10 & 384.53 & 278.76 & 303.68 \\
\hline 10 & 177.95 & 560.40 & 462.28 & 335.22 & 365.18 \\
\hline 20 & 264.30 & 658.40 & 536.70 & 389.16 & 423.96 \\
\hline 25 & 284.91 & 682.70 & 555.82 & 402.83 & 438.90 \\
\hline
\end{tabular}




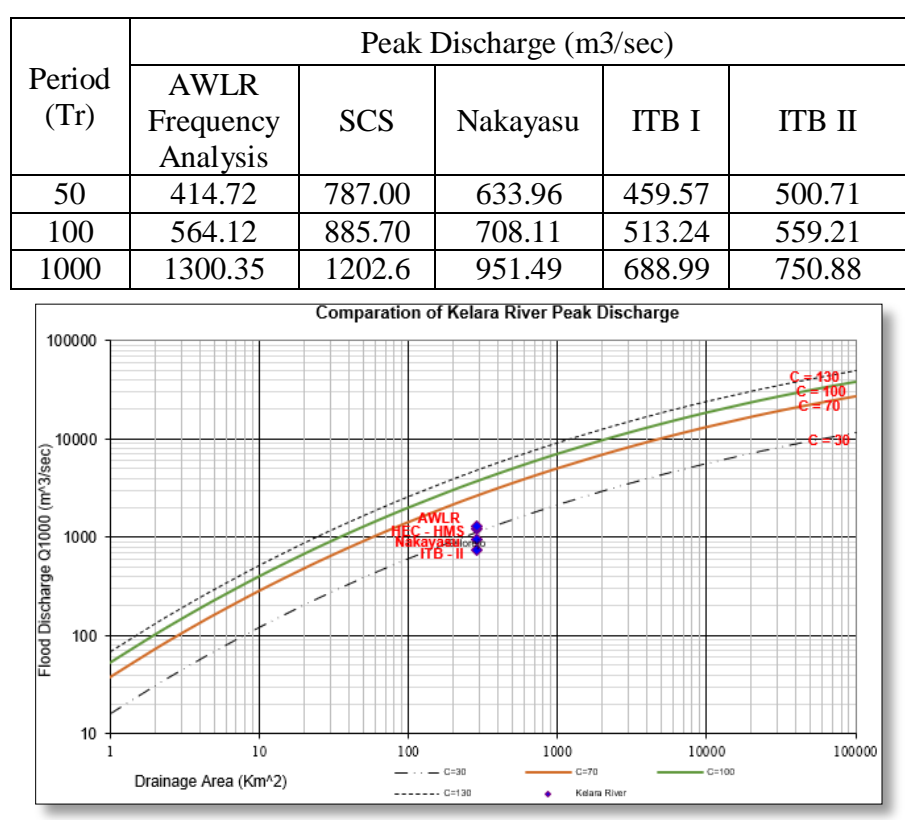

Figure 10. Creager Diagram Kelara Watershed

\section{Conclusion}

According to data analysis result and discussion, it can be concluded that the calculation of design flood discharge according to rainfall data using Synthetic Unit Hydrograph of Nakayasu, ITB I, ITB II, and SCS (HECHMS), and it is known from calculation of design flood discharge that the value of design flood discharge which is the closest to design flood discharge with measured discharge rate and 1000-years Creager value is SCS method. Flood discharge obtained according to SCS method using HEC-HMS 4.8 software application is 2years period is $322,70 \mathrm{~m}^{3} / \mathrm{sec}$, 5-years period is 464,10 $\mathrm{m}^{3} / \mathrm{sec}, 10$-years period is $560,40 \mathrm{~m}^{3} / \mathrm{sec}, 20$-years period is $658,40 \mathrm{~m}^{3} / \mathrm{sec}, 25$-years period is $682,70 \mathrm{~m}^{3} / \mathrm{sec}, 50$ years period is $787,00 \mathrm{~m}^{3} / \mathrm{sec}, 100$-years period is 885,70 $\mathrm{m}^{3} / \mathrm{sec}$, and 1000 -years period is $1202,60 \mathrm{~m}^{3} / \mathrm{sec}$.

\section{References}

[1] Soewarno, Hidrologi, "Aplikasi Metode Statistik Untuk Analisa Data”, (Application of Statistical Methods for Data Analysis), 1st ed. Bandung: Indonesia, : NV, 1995, ch. 2, sec. 1, pp. 1-2.

[2] Y.H.M. Yusuf, "Analisis Hidrologi Sungai Ponre-Ponre Kabupaten Bone", (Hydrological Analysis of the Ponre-Ponre River, Bone Regency), JSPF, vol. 14, pp. 74-80, April 2018.E.M. Wilson, "Hidrologi Teknik", (Engineering Hydrology), 4th ed. Bandung: Indonesia, : ITB, 1990, ch. 1, sec. 4, pp. 4-5.
[3] F.J. Fachri, "Analisis Hidrograf Satuan Sintetis (HSS) Pada Daerah Aliran Sungai (DAS) Jeneberang Kabupaten Gowa", Synthetic Unit Hydrograph Analysis (HSS) in the Jeneberang Watershed (DAS) Gowa Regency), M.S. thesis, Sarjana, UNHAS, Makassar, Indonesia, 2016.

[4] R. Karamma and M.S. Pallu, "Comparison of Model Hidrograf Synthetic Unit (HSS) with the Model of Hydrograf Observation on DAS Jeneberang Gowa Regency, Indonesia," IJISRT, vol. 3, pp. 617-623, February 2018.

[5] N.K. Sari and P. Irawan, "Penerapan Metode Empiris di DAS Batang Lembang untuk Perhitungan Debit Banjir Rancangan”, (Application of Empirical Methods in the Batang Lembang Watershed for Calculation of Design Flood Discharge), AKSELERASI, vol. 2, pp. 52-60, February 2021.

[6] A. Sarminingsih, "Pemilihan Metode Analisis Debit Banjir Rancangan Embung Coyo Kabupaten Grobongan”, (Selection of the Flood Discharge Analysis Method for the Design of the Koto Reservoir, Grobogan Regency), PRESIPITASI, vol. 15, pp. 5361, Maret 2018.

[7] U.S. Lestari, "Kajian Metode Empiris Untuk Menghitung Debit Banjir Sungai Negara di Ruas Kecamatan Sungai Pandan (Alabio)", (Kajian Metode Empiris Untuk Menghitung Debit Banjir Sungai Negara Di Ruas Kecamatan Sungai Pandan (Alabio)), POROS TEKNIK, vol. 8, pp. 86-96, Desember 2016.

[8] J.A. Robot, T. Mananoma, E. Wuisan, and H. Tangkudung "Analisis Debit Banjir Sungai Ranoyapo Menggunakan Metode HSS Gama-I dan HSS Limantara", (Analysis of the Ranoyapo River Flood Discharge Using the HSS Gama-I and HSS Limantara Methods), SIPIL STATIK, vol. 2, pp. 1-12, Januari 2014.

[9] R. Karamma and A.S. Sukri, "Kajian Koefisien Aliran Terhadap Perubahan Debit Banjir DAS Karalloe", (Study of the Flow Coefficient of Changes in Flood Discharge in the Karalloe River Basin), SEMANTIK, vol. 6, pp. 1-8, Januari 2020.

[10] F. Maricar and S.Harto Br "Analisis Kepekaan Hidrograf Satuan Sintetik Gama I dalam Penentuan Debit Banjir Rancangan", (Sensitivity Analysis of Synthetic Gama Unit Hydrographs in Determining Design Flood Discharge), FORUM TEKNIK, vol. 25, pp. 315-328, November 2001.

[11] A.R. Suleman, H. Djufri, M.R. Mustamin, and M. Lawa "Structural Mitigation of Bila River, Sidrap Regency," TURCOMAT, vol. 12, pp. 3573-3577, May 2021.

[12] B. Triatmodjo, "Hidrologi Terapan", (Applied Hydrology), 4th ed. Yogyakarta, Indonesia: BO, 2014, ch. 7, sec. 7, pp. 238-256.

[13] I.M. Kamiana, "Teknik Perhitungan Debit Rencana Bangunan Air", (Water Building Plan Discharge Calculation Technique), 1st ed. Yogyakarta, Indonesia: GI, 2011, ch. 5, sec. 6, pp. 124-125.

[14] Suripin, "Sistem Drainase Perkotaan Yang Berkelanjutan", (Sustainable Urban Drainage System), 1st ed. Yogyakarta, Indonesia: AO, 2004, ch. 2 , sec. 3, pp. 66-67.

[15] D.K. Natakusumah, W. Hatmoko, and D. Harlan, "Prosedur Umum Perhitungan Hidrograf Satuan Sintetis dengan Cara ITB dan Beberapa Contoh Penerapannya", (General Procedure for Calculation of Synthetic Unit Hydrograph Using ITB Method and Some Examples of Its Application), TEKNIK SIPIL, vol. 18, pp. 251-291, Desember 2011

[16] D.T. Pratiwi, “Analisis Hidrograf Menggunakan HEC-HMS (Studi Kasus : DAS Citarum Hulu)", (Hydrograph Analysis Using HEC-HMS (Case Study: Upper Citarum Watershed)), M.S. thesis, Sarjana, IPB, Bogor, Indonesia, 2011.

[17] S. Sosrodarsono, K. Takeda, "Bendungan Type Urugan", (Dam Type Urugan), 5th ed. Jakarta, Indonesia: PP, 2002, ch. 2, sec. 3, pp. 27-45. 